\title{
Um olhar sobre os livros didáticos de álgebra linear à luz da teoria dos registros de representação semiótica
}

\author{
A look at the linear algebra's textbooks in the light of the theory of representation of \\ records semiotics
}

\author{
Valdinei Cezar Cardoso \\ v13dinei@gmail.com \\ Samuel Rocha de Oliveira \\ samrochaoliv@gmail.com \\ Lilian Akemi Kato \\ lilianakemikato@gmail.com
}

\begin{abstract}
Resumo
Este artigo apresenta os resultados de uma pesquisa acerca dos principais livros didáticos de Álgebra Linear, adotados nos cursos de graduação em Matemática, utilizando como lente a Teoria dos Registros de Representação Semiótica de Raymond Duval. Nosso objetivo foi investigar os tipos de representações mais registrados e os tratamentos ou conversões nos exercícios resolvidos dos quatro livros escolhidos. Os resultados indicam que o sistema de registro de representação simbólico-algébrico é o mais utilizado e que os do tipo simbólico-matricial, geométrico-figural ou gráfico são os menos utilizados. Em relação aos tratamentos e as conversões apresentados nas resoluções dos exercícios, notamos que a maioria é resolvida com operações de tratamentos. Dentre as implicações desses resultados destacamos que é preciso propor tarefas que fixem a representação e variem o conteúdo e vice-versa, analisando sempre a relação entre o conteúdo matemático da representação e a própria representação.
\end{abstract}

Palavras-Chave: Álgebra Linear; Livros didáticos; Tratamentos; Conversões; Registros de Representação.

\begin{abstract}
This article presents the results of a survey on the main textbook Linear Algebra, adopted in undergraduate courses in mathematics, using as lens the Theory of Semiotics Representation Registers of Raymond Duval. Our objective was to investigate the types of representations and recorded more treatments or conversions in the solved exercises of the four chosen books. The results indicate that the symbolic-algebraic representation registration system is the most used and that the symbolic-matrix type, geometric, figural or graphic are the least used. Regarding treatments and conversions presented in the resolution of exercises, we note that most are resolved with treatment operations. Among the implications of these results we highlight that it is necessary to propose tasks that fix the representation and vary the content and vice versa, always analyzing the relationship between the mathematical content of the representation and the representation itself.
\end{abstract}

Key Words: Linear Algebra; Didatic books; Treatments; Conversions; Representation records.

\section{Introdução}

Os professores de Matemática planejam grande parte de suas aulas com base no que os livros didáticos apresentam. Segundo o Guia de Livros Didáticos do Plano Nacional do Livro Didático 2014 - PNLD 2014 “o livro didático é um interlocutor que dialoga com o professor e 
com o aluno. Nesse diálogo, o livro é portador de uma perspectiva sobre o saber a ser estudado e sobre o modo mais eficaz de aprendê-lo" (BRASIL, 2013, p. 12).

Nesse sentido, pode-se considerar que a forma como os estudantes têm contato com o conhecimento matemático está fortemente relacionada ao modo como os autores de tais livros apresentam o conhecimento matemático.

Os trabalhos que buscam compreender como o conhecimento matemático é apresentado e organizado nos livros didáticos são importantes para compreendermos algumas dificuldades na aprendizagem de Matemática.

$\mathrm{Na}$ literatura existem trabalhos que analisam como alguns livros de Álgebra Linear (AL) abordam os conteúdos eleitos para o Ensino Superior (de graduação). Entre eles, destacamos o de Karrer (2006), que analisou como quatro livros de AL, adotados em uma amostra de 12 universidades brasileiras, tratam o conceito de transformação linear e o de Grande e Bianchini (2006), que analisou como cinco livros de AL tratam o tema dependência e independência linear. Ambos os trabalhos usaram a ótica dos registros de representação semiótica de Duval (1993).

A Teoria dos Registros de Representação Semiótica (TRRS) de Raymond Duval é uma teoria cognitiva que busca compreender como se dá o aprendizado de conceitos matemáticos, levando em consideração a forma de representação de tais conceitos nas situações de ensino.

Neste trabalho, investigaremos, por meio das lentes dos registros de representação semiótica de Duval (2011), como quatro livros de AL, adotados em cursos de graduação em Matemática da maioria das universidades públicas do Estado do Paraná, tratam os principais conceitos desse campo de estudos, levando em conta os tipos de registros de representação, os tratamentos e as conversões.

Acreditamos que a forma como os conteúdos são apresentados nos livros didáticos influencia na aprendizagem dos conteúdos neles presentes, ainda que na maioria dos cursos de AL haja a mediação de professores com o uso de tais materiais didáticos. Isto é, conjecturamos que a apresentação dos conteúdos nos livros seja determinante no aprendizado dos conteúdos pelos estudantes. Mais estudos serão necessários para verificarmos tal essa afirmação.

Por isso é fundamental saber como os conteúdos relacionados à AL são apresentados nos livros didáticos de $\mathrm{AL}$; assim, neste trabalho, buscaremos responder às seguintes questões: Quais registros de representação são mais utilizados nos exercícios resolvidos dos 
livros? Quais tratamentos ${ }^{1}$ e conversões $^{2}$ de registros de representação são explorados nos enunciados e nas resoluções dos exercícios?

Escolhemos investigar os exercícios resolvidos, pelo fato de os estudantes buscarem com frequência os exemplos resolvidos nos livros como ponto de partida para a resolução de atividades extraclasse propostas pelos professores. A busca pelos tratamentos e pelas conversões, utilizadas em tais obras, foi motivada pela conclusão de Duval (2009) de que a aprendizagem matemática depende da capacidade dos estudantes reconhecerem um mesmo objeto matemático em mais de um registro de representação semiótica.

\section{A Teoria das Representações Semióticas}

Para Duval (2009, p. 29) o acesso aos objetos matemáticos ${ }^{3}$ se dá por meio de suas representações e "não há conhecimento que não possa ser mobilizado por um sujeito sem uma atividade de representação".

Duval (2006) lembra que há três tipos de representações: as mentais - compostas pelo conjunto de concepções de um indivíduo; as semióticas - que exteriorizam as representações mentais do indivíduo, são externas, conscientes e seriam percepções externas que foram interiorizadas; por fim, as representações internas ou computacionais - são aquelas cuja execução é automática quando o indivíduo realiza determinada tarefa.

Sendo estas últimas, relacionadas a um sistema particular de signos (a linguagem, a escrita, os gráficos cartesianos, as figuras, os sistemas numéricos, a escrita algébrica) que podem ser convertidos em representações "equivalentes" em outro sistema de registros de representação semiótica. De acordo com França (2007, p. 14)

Duval expressa questões relativas à aprendizagem matemática, relacionando os processos de semiosis e noesis. Entende-se por semiosis a apreensão ou a produção de uma representação semiótica - ou ainda, uma representação por meio de signos - e, por noesis, os atos cognitivos, como a apreensão conceitual de um objeto, a discriminação de uma diferença ou a compreensão de uma inferência - em suma, a aquisição conceitual. De acordo com esse pesquisador, não há aquisição conceitual (conhecimento) de um objeto sem recorrer a sistemas semióticos (representações). Em outras palavras, não existe noesis sem semiosis. Com isso, as representações mentais e as representações semióticas não podem ser dissociadas, ao contrário, há uma estreita interdependência entre elas, de forma que, para garantir o primeiro passo na direção da noesis, é necessária a semiosis.

\footnotetext{
${ }^{1}$ Transformações internas realizadas dentro de um mesmo registro de representação semiótica. Como exemplo de tratamento, podemos citar a resolução de uma equação do primeiro grau.

2 Transformações de representações de um registro de representação semiótica para um outro registro de representação. Por exemplo, a passagem da representação da função polinomial $f(x)=x+1$ para a sua representação gráfica, num sistema de coordenadas.

${ }^{3}$ Como exemplos de objetos matemáticos, podemos citar: um cubo, uma pirâmide, uma transformação linear,
} etc. 
Para Duval (2003, p. 16) ao estudar Matemática os sujeitos são confrontados com situações em que têm que fazer "transformações de representações dentro de um mesmo registro, por exemplo: efetuar um cálculo ficando estritamente no mesmo sistema de escrita ou de representação" (tratamentos) ou em alguns casos, precisam desenvolver conversões, que seriam:

\footnotetext{
"transformações de representação que consistem em mudança de registro conservando os mesmos objetos denotados: por exemplo, reconhecer a escrita algébrica de uma equação em sua representação gráfica".
}

Para isso, é necessário, mas não suficiente, detectar os registros de representação semiótica que os estudantes explicitam durante o estudo da Matemática. Tais registros podem ser expressos nas formas: tabular, língua natural, gráfica, figural, algébrica, entre outras.

Para analisar a aprendizagem da Matemática, é preciso considerar todos os registros mobilizados em atividades matemáticas, utilizados para o desenvolvimento de uma determinada tarefa, mais especificamente, as conversões entre eles, que ficam implícitas, mas que devem se tornar espontâneas.

Por isso, acreditamos que, ao detectarmos as transformações mais utilizadas nos livros didáticos de $\mathrm{AL}$, podemos compreender um pouco mais, as possíveis influências do seu uso na aprendizagem conceitual dos estudantes.

Nesse sentido, inferimos que, se os livros didáticos de AL apresentarem, nos exercícios resolvidos, múltiplas representações de um mesmo registro então, com a esta experiência, os estudantes poderão ter menos dificuldades ao realizar conversões ou tratamentos com esses registros. Na próxima seção, vamos investigar se os livros de AL analisados neste trabalho privilegiam as conversões ou os tratamentos.

\section{Analisando alguns livros de AL}

Afim de, conhecermos quais os livros de AL adotados nos 21 cursos de Licenciatura em Matemática presenciais das universidades públicas do Estado do Paraná, enviamos um email para os coordenadores destes cursos, informando sobre a nossa investigação e solicitando o plano de ensino da disciplina de Álgebra Linear oferecida para estes cursos, recebemos a resposta de 10 coordenadores de cursos (Quadro 2).

Consultando os referidos planos de ensino identificamos os quatro livros mais citados, os quais foram denominados por L1, L2, L3 e L4. Nestas obras, analisamos os exercícios resolvidos buscando identificar se, em cada um deles, o autor efetuava tratamentos ou conversões de registros de representação semiótica, considerando os seus enunciados e as suas resoluções. 
Quadro 1: Livros selecionados para a nossa análise

\begin{tabular}{|l|l|}
\hline Livro & \multicolumn{1}{|c|}{ Descrição } \\
\hline L1 & Álgebra Linear. José Luiz Boldrini [et al.]. 3 ed. 1980, Harper \&Row do Brasil. \\
\hline L2 & Álgebra Linear. Elon Lages Lima. 2 ${ }^{\text {a Edição, 1996, IMPA. }}$ \\
\hline L3 & $\begin{array}{l}\text { Álgebra Linear e Aplicações. Carlos A. Callioli; H. H. Domingues; Roberto C. F. Costa. } 5^{\text {a }} \text { Edição, 1987, } \\
\text { Atual Editora. }\end{array}$ \\
\hline L4 & Álgebra Linear. Alfredo Steinbruch, Paulo Winterle. 2 ed. 1987, Pearson Makron Books. \\
\hline
\end{tabular}

Fonte: Programas da disciplina de AL das universidades selecionadas.

O Quadro 2 mostra quais dos livros indicados no Quadro 1 fazem parte dos programas de AL dos cursos de Licenciatura em Matemática integrantes desta pesquisa.

Quadro 2: Algumas universidades públicas paranaenses que usam os livros indicados anteriormente

\begin{tabular}{|l|l|l|l|c|c|}
\hline \multicolumn{1}{|c|}{ Instituição de Ensino superior } & \multicolumn{1}{|c|}{ Sigla } & L1 & L2 & L3 & L4 \\
\hline Universidade Estadual de Londrina & UEL & & $\mathrm{x}$ & & $\mathrm{x}$ \\
\hline Universidade Estadual de Maringá & UEM & $\mathrm{x}$ & $\mathrm{x}$ & $\mathrm{x}$ & $\mathrm{x}$ \\
\hline Universidade Federal do Paraná & UFPR & $\mathrm{x}$ & & & \\
\hline Universidade Estadual do Paraná (Apucarana) & UNESPAR & $\mathrm{x}$ & $\mathrm{x}$ & $\mathrm{x}$ & $\mathrm{x}$ \\
\hline Universidade Estadual do Paraná (Campo Mourão) & UNESPAR & $\mathrm{x}$ & $\mathrm{x}$ & & $\mathrm{x}$ \\
\hline Universidade Estadual do Paraná (Paranavaí) & UNESPAR & $\mathrm{x}$ & $\mathrm{x}$ & & $\mathrm{x}$ \\
\hline Universidade Estadual do Paraná (União da Vitória) & UNESPAR & $\mathrm{x}$ & $\mathrm{x}$ & & $\mathrm{x}$ \\
\hline Universidade Estadual do Centro-Oeste & UNICENTRO & $\mathrm{x}$ & & & $\mathrm{x}$ \\
\hline Universidade Tecnológica Federal do Paraná (Toledo) & UTFPR & $\mathrm{x}$ & $\mathrm{x}$ & $\mathrm{x}$ & \\
\hline Universidade Estadual do Norte do Paraná (Cornélio Procópio) & UENP & $\mathrm{x}$ & $\mathrm{x}$ & & $\mathrm{x}$ \\
\hline
\end{tabular}

Fonte: Planos de ensino da disciplina de AL para o curso de Licenciatura em Matemática.

O Quadro 2 mostra que nove cursos adotam L1, oito adotam L2 ou L4 e três adotam L3. A análise dos exercícios resolvidos dos quatro livros se deu à luz dos registros de representação semiótica utilizados nos enunciados dos problemas e nas suas resoluções, que foram organizados em categorias (Quadro 3) já apresentadas nos trabalhos de Grande e Bianchini (2006), Karrer (2006) e Duval (2003, 2006).

\section{Resultados}

O Quadro 3 apresenta os tipos de registros de representação que identificamos nos livros didáticos analisados e a sigla utilizada para cada um deles. 
Quadro 3: Tipos de registros detectados nas soluções dos exercícios resolvidos de cada livro

\begin{tabular}{|l|l|}
\hline \multicolumn{1}{|c|}{ Registros } & \multicolumn{1}{c|}{ Sigla } \\
\hline Simbólico algébrico & S.A. \\
\hline Simbólico matricial & SM \\
\hline Numérica por n-uplas & NN \\
\hline Numérica matricial & NM \\
\hline Figural & F \\
\hline Gráfica & G \\
\hline $\begin{array}{l}\text { Linguagem natural } \\
\text { especializada }\end{array}$ & LN \\
\hline
\end{tabular}

Fonte: Karrer (2006, Adaptado)

Após a definição dos tipos de registros de representação que iríamos procurar em cada um dos enunciados (Registros de partida (RP)) e resoluções (Registros de Chegada (RC)) dos exercícios resolvidos presentes nos referidos livros, identificamos e quantificamos os exercícios que utilizaram tratamentos e os que realizavam conversões de registros de representação semiótica.

Tal levantamento originou o Quadro 4, onde, por questões estéticas, denotamos as conversões pela letra "C", os tratamentos pela letra " $\mathrm{T}$ ” e o número de exercícios resolvidos analisados em cada livro pela letra "E".

Quadro 4: Tratamentos ou conversões de registros apresentados em cada livro

\begin{tabular}{|c|c|r|r|r|r|r|r|r|r|r|r|r|r|r|}
\hline \multicolumn{2}{|c|}{ Registro } & \multicolumn{3}{|c|}{ L1 } & \multicolumn{3}{c|}{ L2 } & \multicolumn{3}{c|}{ L3 } & \multicolumn{3}{c|}{ L4 } & \\
\hline SAÍDA & CHEGADA & T & C & E & T & C & E & T & C & E & T & C & E & Total \\
\hline F & S.A. & 0 & 0 & 0 & 0 & 0 & 0 & 0 & 0 & 0 & 0 & 0 & 0 & 0 \\
\hline F & LN & 0 & 3 & 3 & 0 & 0 & 0 & 0 & 0 & 0 & 0 & 0 & 0 & 3 \\
\hline LN & LN & 0 & 0 & 0 & 54 & 0 & 54 & 23 & 0 & 23 & 0 & 0 & 0 & 77 \\
\hline LN & NM & 0 & 0 & 0 & 0 & 0 & 0 & 0 & 0 & 0 & 0 & 0 & 0 & 0 \\
\hline LN & S.A. & 0 & 10 & 10 & 0 & 0 & 0 & 0 & 0 & 0 & 0 & 0 & 0 & 10 \\
\hline LN & NN & 0 & 0 & 0 & 0 & 0 & 0 & 0 & 2 & 2 & 0 & 6 & 6 & 8 \\
\hline LN & F & 0 & 6 & 6 & 0 & 0 & 0 & 0 & 0 & 0 & 0 & 0 & 0 & 6 \\
\hline NM & S.A. & 0 & 7 & 7 & 0 & 1 & 1 & 0 & 5 & 5 & 0 & 1 & 1 & 14 \\
\hline NM & NN & 0 & 4 & 4 & 0 & 0 & 0 & 0 & 0 & 0 & 0 & 0 & 0 & 4 \\
\hline NM & NM & 17 & 0 & 17 & 4 & 0 & 4 & 26 & 0 & 26 & 27 & 0 & 27 & 74 \\
\hline NN & S.A. & 0 & 3 & 3 & 0 & 0 & 0 & 0 & 2 & 2 & 0 & 4 & 4 & 9 \\
\hline NN & NM & 0 & 5 & 5 & 0 & 3 & 3 & 0 & 5 & 5 & 0 & 4 & 4 & 17 \\
\hline NN & NN & 9 & 0 & 9 & 1 & 0 & 1 & 9 & 0 & 9 & 19 & 5 & 24 & 43 \\
\hline NN & LN & 0 & 0 & 0 & 0 & 0 & 0 & 0 & 0 & 0 & 0 & 2 & 2 & 2 \\
\hline NN & F & 0 & 1 & 1 & 0 & 0 & 0 & 0 & 0 & 0 & 0 & 0 & 0 & 1 \\
\hline
\end{tabular}




\begin{tabular}{|l|c|r|r|r|r|r|r|r|r|r|r|r|r|r|} 
S.A. & G & 0 & 11 & 11 & 0 & 0 & 0 & 0 & 2 & 2 & 0 & 13 & 13 & 26 \\
\hline S.A. & NM & 0 & 12 & 12 & 0 & 4 & 4 & 0 & 18 & 18 & 0 & 12 & 12 & 46 \\
\hline S.A. & NN & 0 & 3 & 3 & 0 & 1 & 1 & 0 & 3 & 3 & 0 & 4 & 4 & 11 \\
\hline S.A. & SM & 0 & 1 & 1 & 0 & 0 & 0 & 0 & 0 & 0 & 0 & 0 & 0 & 1 \\
\hline S.A. & S.A. & 25 & 0 & 25 & 22 & 0 & 22 & 112 & 0 & 112 & 30 & 0 & 30 & 189 \\
\hline S.A. & F & 0 & 3 & 3 & 0 & 1 & 1 & 0 & 0 & 0 & 0 & 0 & 0 & 4 \\
\hline SM & S.A. & 0 & 3 & 3 & 0 & 0 & 0 & 0 & 0 & 0 & 0 & 1 & 1 & 4 \\
\hline SM & SM & 1 & 0 & 1 & 1 & 0 & 1 & 7 & 0 & 7 & 2 & 0 & 2 & 11 \\
\hline \multicolumn{1}{l}{ Total } & 52 & 72 & 124 & 82 & 10 & 92 & 177 & 37 & 214 & 78 & 52 & 130 & 560 \\
\hline
\end{tabular}

Fonte: Exercícios resolvidos dos livros L1 até L4.

Como apresenta o quadro anterior, o livro L3 é o que traz o maior número de exercícios resolvidos (214). O livro L2, que apresenta 92 exercícios resolvidos e foi adotado por oito universidades.

A análise do Quadro 4 mostra que aproximadamente $70 \%$ dos 560 exercícios resolvidos analisados foram resolvidos por meio de tratamentos de registros de representação semiótica, isto é, tinham o mesmo registro no enunciado e também na resolução dos problemas propostos.

Os registros de representação utilizados nos tratamentos ficaram assim distribuídos: $48 \%$ com os registros S.A., $20 \%$ com $\mathrm{LN}$ e NM cada, $10 \%$ com NN e $2 \%$ com SM.

O fato dos livros utilizarem conversões de registros de representações em apenas $30 \%$ dos exercícios resolvidos pode contribuir para o aparecimento de dúvidas por parte dos estudantes, nas situações em que precisam efetuar uma conversão de registros, já que, segundo Duval (2011), só é possível ter indícios de aprendizagem de um determinado conceito quando o sujeito consegue identificá-lo em, pelo menos, dois sistemas de registros de representação diferentes.

O livro L1 destacou-se em relação aos demais, por apresentar 72 exercícios resolvidos, nos quais os autores utilizam conversões de registros de representação, o que corresponde a aproximadamente $58 \%$ dos seus exercícios resolvidos. E, como o livro faz parte do programa da disciplina de AL da maioria dos programas de AL analisados, inferimos que a escolha de tais cursos foi acertada, do ponto de vista da TRRS, pois o fato de um livro desenvolver exercícios utilizando diferentes registros de representação, pode favorecer a aprendizagem dos estudantes.

O Quadro 4 apresenta os registros de partida e de chegada de todos os exercícios resolvidos dos livros L1 até L4. Eles nos dão um panorama geral dos tipos de registros 
utilizados nos enunciados e nas resoluções dos exercícios resolvidos apresentados, indicando o número de tratamentos $(\mathrm{T})$ ou conversões $(\mathrm{C})$ detectados.

Como dissemos anteriormente, a análise dos exercícios resolvidos, presentes nestes livros, nos permitiu inferir que a maioria deles apresenta tratamentos de registros de representação semiótica (aproximadamente 70\%). Para compreendermos um pouco mais as características dos exercícios resolvidos, discutiremos brevemente sobre as características e os sistemas de registros de representação utilizados pelos autores destas obras para desenvolverem os exercícios resolvidos em cada uma delas.

\subsection{Registro: simbólico}

\subsubsection{Algébrico}

O Quadro 4 mostra que a maioria dos exercícios resolvidos utiliza os registros do tipo S.A., tanto no enunciado (RP) como na resolução (RC). Percebe-se que os autores de todos os livros analisados privilegiam os tratamentos, apresentando poucas resoluções que exigem conversões.

Para Duval (1995, apud BRANDITT, MORETTI, 2013, p. 58),

$$
\begin{aligned}
& \text { ao privilegiar o tratamento, estar-se-á reforçando a importância da forma, admitindo } \\
& \text { que ela seja responsável pela descrição de uma informação. No entanto, a operação } \\
& \text { cognitiva de conversão envolve uma mudança de forma que é importante por } \\
& \text { evidenciar: resposta ao conteúdo representado, possibilidade de uma diversidade das } \\
& \text { formas de representação para um mesmo conteúdo representado ou possibilidade por } \\
& \text { uma mudança das formas de representação por razões de economia de tratamento, a } \\
& \text { complementaridade de registros e a conceitualização que implica a coordenação de } \\
& \text { registros de representação. Ela tem que ser privilegiada, visto não ser nem trivial } \\
& \text { nem cognitivamente neutra e permitir a diferenciação entre representante e } \\
& \text { representado. }
\end{aligned}
$$

Uma característica dos exercícios cujos enunciados são do tipo S.A. é que a escrita algébrica, ao mesmo tempo em que permite a síntese de um grande número de ideias com um pequeno número de símbolos, pode também exigir dos estudantes alguns conhecimentos prévios que, muitas vezes, eles não possuem.

Gagné (1985) afirma que a natureza do conhecimento a ser aprendido pode gerar dificuldades para a aprendizagem, pois cada tipo de representação traz consigo algumas especificidades que podem acarretar algumas dificuldades de compreensão.

Os exercícios que têm os enunciados predominantemente com os registros S.A. e as resoluções com os registros S.A. aparecem em todos os assuntos tratados pelos livros analisados. Durante a resolução de tais exercícios, os autores privilegiam os tratamentos, 
utilizando manipulações algébricas e as propriedades dos conceitos tratados, para esboçarem as soluções.

Machado (2011, p. 38) ainda afirma que

para a construção de uma demonstração, deve-se selecionar e explicitar as evidências elementares que constituirão o ponto de partida necessário e que dependerão, fundamentalmente, do canal de comunicação que vier a ser estabelecido entre o emissor e o receptor da mensagem.

Os livros L1, L3 e L4 apresentam, em seus exercícios resolvidos, conversões que partem do registro S.A. e chegam ao registro de representação G. Tais conversões foram utilizadas predominantemente quando o tema estudado era transformação linear.

Não detectamos exercícios nos quais o enunciado apresentasse um gráfico (G) e a resolução fosse, a obtenção da lei de formação (registro S.A.) da transformação linear. Detectamos apenas exercícios resolvidos que partiam da lei de formação e chegavam ao registro gráfico.

Como relatamos anteriormente, nossa análise mostrou que os livros de AL analisados neste trabalho expressam o conteúdo matemático predominantemente na forma algébrica, o que pode indicar uma tentativa dos autores de simplificar a apresentação de conceitos que, se fossem apresentados somente com o uso da língua materna, demandariam um grande volume de páginas para a sua discussão.

Machado (2011) e Gagné (1985) defendem que a maioria dos conceitos matemáticos, entre eles, aqueles envolvidos na AL, é desprovida de um significado prontamente perceptível apenas pela observação da sua representação, seja ela figural ou algébrica ou numérica.

Notamos ainda que as conversões S.A. para NM, apresentadas em todos os livros analisados, são utilizadas, principalmente, quando os autores tratam dos seguintes temas: álgebra matricial, sistemas de equações lineares e matriz de uma transformação linear.

De um modo geral, tais autores iniciam os exercícios com a apresentação do sistema linear a ser resolvido, em seguida escrevem a matriz ampliada correspondente ao sistema, escalonam-na e, por fim convertem-na em um sistema equivalente, que tem o mesmo conjunto solução do sistema apresentado inicialmente, para finalmente obterem a solução. A seguir, discutiremos os exercícios que utilizam os registros de representação SM, como registro de chegada ou de partida.

\subsubsection{Matricial}

A quantidade de tratamentos envolvendo o registro SM, tanto nos enunciados quanto nas resoluções, é pouco privilegiada nos livros analisados: apenas $2 \%$ dos tratamentos 
detectados nos exercícios foram feitos com esse tipo de registro. O livro L3 foi o que apresentou o maior número de exercícios resolvidos, utilizando esse tipo de registro de representação semiótica.

Em relação às conversões de registros de representação envolvendo os registros SM, notamos que L1 apresentou três conversões do tipo SM-S.A. e uma do tipo S.A.-SM, já L4 apresentou uma conversão do tipo SM-S.A.. Como apenas L1 apresentou conversões do tipo SM-S.A. e S.A.-SM, notamos que, de um modo geral, os livros se preocuparam em efetuar conversões de ida e de volta, por exemplo, dos sistema S.A. para o sistema SM e vice-versa.

Acreditamos que o potencial educativo dos livros poderia ser maior se explorassem outros tipos de conversões do sistema SM para outros sistemas e destes para o SM.

Nossa análise permitiu verificar que os conteúdos que mais utilizam os registros SM foram: as operações com matrizes, a matriz de uma transformação linear e os autovalores e autovetores. Nossa prática profissional nos permite inferir que a aplicação rotineira de algoritmos e a resolução de exercícios mecânicos ou de um mesmo tipo são insuficientes para que os estudantes compreendam a natureza lógica e coerente da linguagem matemática.

Pensando em tais aspectos e analisando os exercícios resolvidos dos livros supracitados, inferimos que estes poderiam trazer um número maior de exercícios resolvidos do tipo SM. Assim, os estudantes que os utilizam em seus cursos de graduação poderiam integrar mais registros S.A. com registros SM e isso poderia favorecer a construção de conhecimentos relacionados ao domínio e à manipulação de situações-problemas envolvendo esses dois tipos de registros.

Isso porque, por trás do enunciado e da resolução de um exercício, repousam vários conhecimentos anteriores que os estudantes têm que mobilizar para resolver o problema. Para Sierpinska (2005), a dificuldade na construção do raciocínio algébrico, muitas vezes, originase do fato de as pessoas não utilizarem os símbolos matemáticos em tarefas do seu dia a dia.

$\mathrm{Na}$ próxima subseção, trataremos dos registros de representação semiótica numérico por n-uplas e numérico matricial.

\subsection{Registro numérico}

\subsubsection{Por n-uplas}

Os dados do Quadro 4 indicam que os livros analisados apresentam mais conversões (52 conversões) do que tratamentos (43 tratamentos), envolvendo o registro NN. Tais conversões são distribuídas nos seguintes sistemas de registros de representação semiótica, 
seguidos do número de vezes que foi identificado: NN-NM (17), NM-NN (4), NN-S.A. (9), S.A.-NN (11), LN-NN (8), NN-LN (2) e NN-F (1).

O fato de os livros utilizarem mais conversões do que tratamentos envolvendo o sistema de registros de representação NN é positivo do ponto de vista da TRRS, pois pode ajudar os estudantes que utilizam esses livros a compreenderem um mesmo conceito em mais de um tipo de registro de representação semiótica. Na próxima subseção, abordaremos o sistema de registros de representação semiótica numérico matricial (NM).

\subsubsection{Matricial}

Por meio da nossa análise, notamos que nem todos os livros de AL propõem sugestões de atividades para o ensino de matrizes e, como veremos mais adiante, em muitos casos, os estudantes não se lembraram se estudaram matrizes ou algumas de suas propriedades. Tal cenário pode ser consequência da forma descontextualizada e passiva com que os estudantes vêm aprendendo esse conceito (MESSIAS, SÁ e FONSECA, 2007).

Percebemos que o livro L2 é o único que não traz um capítulo voltado ao estudo das operações com matrizes. Ao iniciar tal livro, o autor já pressupõe que os estudantes de AL dominam os processos envolvidos nas operações com matrizes. Os demais livros apresentam as operações com matrizes e algumas propriedades da álgebra matricial. $\mathrm{Na}$ próxima subseção, trataremos dos registros de representação gráficos e figurais.

\subsection{Registros de Representação Semiótica do tipo Gráfico (G) e do tipo Figural (F)}

Para Machado (2011, p. 48), “os objetos matemáticos, desde os mais simples até as estruturas mais complexas, admitidas ou não as raízes empíricas, são peremptoriamente classificados como abstrações". A representação gráfica de uma função é, assim como a representação algébrica, uma abstração.

Assim, se os estudantes tiverem contato com situações em que são explorados diferentes registros de representação, a possibilidade de que eles compreendam, de forma mais profunda, situações semelhantes e tenham sucesso na sua resolução será ampliada.

Vale lembrar que o uso de diferentes representações para explicar um mesmo conceito só contribui para a aprendizagem dos estudantes se tais representações se complementarem, auxiliando-os a perceber características dos objetos matemáticos que ficam ocultas quando os objetos são estudados, utilizando apenas um tipo de registro de representação. 
Se forem utilizadas de maneira aleatória e em todos os tipos de situações-problemas, a abordagem por meio de diferentes representações pode favorecer o aparecimento de concepções equivocadas e erros conceituais por parte dos estudantes.

Podemos inferir que o uso de diferentes representações deve respeitar o aprendizado dos estudantes e o tempo de cada um, pois aprender um novo conceito, em muitos casos, pode não ser uma tarefa fácil, ainda mais, quando a explicação utiliza duas representações já no primeiro contato do estudante com o tema.

$\mathrm{Na}$ próxima subseção, abordaremos algumas características da representação de conceitos matemáticos, em particular, conceitos de AL, expressos por meio da linguagem natural especializada.

\subsection{Linguagem Natural Especializada}

Em Matemática e, em especial, na AL, grande parte das discussões que envolvem a construção de aprendizagens relacionadas aos conceitos afetos a esta disciplina se inicia por meio da linguagem natural de uso especializado ${ }^{4}$, seja ela escrita nos livros-texto ou falada pelos professores em suas aulas.

Tal forma de expressar conceitos relacionados à AL contribui em alguns aspectos, como, por exemplo, ao usar a língua natural especializada para abordar conceitos específicos dessa disciplina, os professores ou autores de livros aproximam a sua mensagem dos estudantes que dominam a mesma linguagem, de uso comum.

Somente a linguagem natural seria suficiente para representar todos os conceitos envolvidos em AL, mas as explicações ficariam longas. As notações simbólicas da Matemática em geral e da AL em particular têm também o papel de encapsular ou sintetizar longas frases ou explicações em linguagem natural. Se todas as expressões fossem sempre escritas em linguagem natural, os livros didáticos de Matemática seriam muito maiores e tediosos de ler.

Hillel e Dreyfus (2005) constatam que o uso de termos matemáticos por estudantes, muitas vezes, é impreciso. No entanto, tal imprecisão não prejudica o diálogo e a discussão de tais assuntos por estudantes de uma mesma turma ou dos estudantes com o professor.

Vergnaud (1990) defende que equívocos que podem surgir pela falta de experiência em lidar com a linguagem matemática podem ser superados ao longo do tempo com o uso de

\footnotetext{
${ }^{4}$ Em nosso trabalho, sempre que utilizarmos a expressão linguagem natural, estaremos nos referindo à linguagem natural de uso especializado, proposta por Duval (2009).
} 
diferentes situações por um longo período de tempo. Isto se dá, segundo Sierpinska (2005), pelo fato de que uma das vias para o favorecimento da compreensão de conceitos matemáticos seria composta pelas interações e pelas trocas de significados presentes nos diálogos. Tais situações ajudam a moldar a forma como as pessoas constroem o seu repertório de conceitos matemáticos.

Tentando solucionar esse problema, nos últimos anos, diversos pesquisadores (DUVAL, 1995; KIERAN, FORMAN, SFARD, 2001; VERGNAUD, 1990) têm se dedicado ao estudo da linguagem e suas implicações em situações educacionais. Sierpinska (2005, p. 205) faz uma síntese das principais linhas de investigação de tais trabalhos, relatando que a linguagem é entendida, para alguns, como um código; para outros, como um discurso; e para um terceiro grupo, como uma representação.

Durante tal processo, é fundamental o recurso às diferentes representações dos conceitos matemáticos, que podem oportunizar a compreensão de propriedades que não ficariam evidentes se os temas fossem estudados apenas em um sistema de registros de representação semiótica.

Assim, podemos inferir que, mesmo quando a representação utilizada para um conceito de AL é a linguagem natural, é fundamental o papel do professor para mediar a relação entre os estudantes e os livros didáticos. Isso porque, se um tema é estudado por meio da linguagem natural especializada, não significa que seu aprendizado será mais fácil do que se estivesse sendo estudado por meio de outro sistema de registros de representação semiótica. Para Duval (2011), cada tipo de registro (isolado) de representação tem suas particularidades e, consequentemente, pode trazer consigo dificuldades para os aprendizes.

\section{Algumas conclusões}

Em nossa pesquisa identificamos, nos exercícios resolvidos dos livros analisados, expressões do tipo: "não é difícil concluir que..." "Deixamos ao leitor a demonstração dos detalhes..." ou "De forma trivial mostramos que...", "Facilmente pode-se concluir que...", entre outras, que têm a intenção de encorajar o estudante a concluir os resultados mais "simples", do ponto de vista do autor. No entanto, o aprendizado conceitual, em especial o da $\mathrm{AL}$, depende de conhecimentos anteriores que nem sempre foram construídos pelos estudantes.

Desta maneira, se o estudante não compreender a "forma trivial" com que tais resultados são mostrados, ele pode ter um empecilho para o seu aprendizado. 
Daí torna-se importante para os autores buscarem o equilíbrio entre aquilo que deve-se deixar sob a responsabilidade dos estudantes e o detalhamento das resoluções dos exercícios e pode-se, na medida do possível, recapitular conhecimentos anteriores que podem acarretar dúvidas.

Nesse contexto, a apresentação de diferentes representações para um mesmo conceito, dentro de um exercício resolvido no livro, possibilitará, ao estudante, buscar alguns elos, já conhecidos, para a compreensão do problema, principalmente quando destacamos os papéis das conversões de registros de representação nesse processo.

Entretanto, nossa análise mostrou que são raras as conversões de registros de representação em alguns tópicos, como os espaços vetoriais, por exemplo. Diante disso, assumimos que uma das dificuldades para o aprendizado de conceitos matemáticos está na mobilização implícita de vários registros, sempre que o indivíduo necessitar apresentar uma ideia explicitamente.

Para Duval (2011), em Matemática, raramente pensamos em um único registro, mas em vários registros ao mesmo tempo, ainda que as produções privilegiem um único registro, como é o caso dos livros de AL, que, como aponta a literatura, utilizam, predominantemente, o registro simbólico.

Pela especificidade dos conceitos relacionados à $\mathrm{AL}$, verificamos que os tipos de registros de representação semiótica mais utilizados são os registros simbólico-algébricos, e os menos utilizados são os do tipo simbólico-matricial, geométrico-figural e gráfico e do ponto de vista da TRRS, só podemos ter indícios de aprendizagem de um determinado conceito se os estudantes forem capazes de reconhece-los em sob diferentes representações.

Em nossa análise notamos que o fato de os livros priorizarem um determinado sistema de registros e não utilizarem outros, indica que, em geral, os livros não procuram apresentar um mesmo conceito sob diferentes representações, o que pode aumentar dificuldades de aprendizagem em AL.

Sobre os tratamentos e as conversões apresentados nas resoluções dos exercícios, notamos que a maioria é resolvida com operações de tratamentos. Nesse tipo de procedimento, os autores realizam operações e desenvolvem algoritmos para a resolução dos exercícios, sem mudar o tipo de registro de representação semiótica. Isto é, a maioria dos exercícios apresentados não oportuniza aos estudantes o contato com diferentes representações semióticas, relacionadas a um mesmo conceito, o que para Duval (2006) impossibilita os professores de saberem se os estudantes compreenderam os conceitos 
estudados, já que a maioria dos conceitos é apresentada apenas por um único sistema de registros de representação.

Segundo Duval (2011), o aprendizado da Matemática se dá pela mobilização de, pelo menos, dois tipos de registros de representação. Nesse sentido, acreditamos que o fato de a maioria dos livros analisados priorizar os tratamentos pode prejudicar o aprendizado conceitual dos estudantes.

Além disso, Duval (2006) lembra que toda atividade matemática reúne, por um lado, o conteúdo conceitual e não semiótico do objeto matemático e, por outro, representações semióticas que são escolhidas, de acordo com o contexto e o problema de estudo. Para ele, a pedra fundamental da compreensão conceitual é a capacidade de transferir conhecimentos de contextos matemáticos para contextos não matemáticos. Para isso, é fundamental compreender os objetos matemáticos em diferentes contextos e sob diferentes representações.

Em todos os livros analisados, notamos que o tema transformações lineares é o que possibilitou o maior número de conversões de registros de representação semiótica, tendo sido as principais conversões utilizadas as do registro de representação simbólico-algébrico para o gráfico. Inferimos que os autores poderiam também apresentar outros tipos de conversões como por exemplo: do registro gráfico para o simbólico-algébrico, o que poderia potencializar a aprendizagem dos estudantes acerca deste tema, já que realizariam conversões partindo de sistemas de registros diferentes e assim teriam a oportunidade de conhecer as propriedades das transformações lineares, partindo de diferentes registros de representação.

No entanto, Duval (2006, p. 162) ressalta que não basta que os autores coloquem em seus livros múltiplas representações de um mesmo conceito, utilizando diferentes tratamentos ou conversões de registros. É preciso propor tarefas que fixem a representação e variem o conteúdo e vice-versa, analisando sempre a relação entre o conteúdo matemático da representação e a própria representação.

Por fim, os livros analisados são adotados em cursos de licenciatura em Matemática de universidades públicas do Estado do Paraná, que tem entre o seu quadro docente, profissionais titulados nas mais diversas universidades públicas do Brasil e do exterior e que são eles que definem as referências bibliográficas adotadas na disciplina de AL de cada uma destas instituições. Entendemos também que os resultados apontados em nosso trabalho estão em consonância com o que ocorre em outras universidades brasileiras. 


\section{Referências:}

BOLDRINI, J. L. et al.. Álgebra Linear. São Paulo: Harper \& Row do Brasil, 1980.

BRANDITT, C. F.; MORETTI, M. T. Aprendizagem do sistema de numeração. 1 ed. Curitiba: Prismas, 2013.

BRASIL. Guia de livros didáticos: PNLD 2014: matemática. Brasília: Ministério da Educação, Secretaria de Educação Básica, 2013.

CAlliOli, C. A.; DOMINGUES, R. C. F.; COSTA, R. C. F.. Álgebra Linear e Aplicações. 5a Ed.. São Paulo: Atual Editora, 1987.

DUVAL, R. Registres de répresentation sémiotique et fonctionnement cognitif de la pensée. Annales de Didactique et des Sciences Cognitives, IREM de Strarsbourg v.5, p.37-65, 1993.

DUVAL, R.. Sémiosis et pensée humaine: registres sémiotiques et apprentissages intellectuels. Paris: Peter Lang, 1995.

DUVAL, R.. Registros de Representações Semióticas e Funcionamento Cognitivo da Compreensão em Matemática. In: MACHADO, Silvia D. A. Aprendizagem em Matemática: Registros de Representação Semiótica. Campinas: Editora Papirus, p.11-34, 2003.

DUVAL, R. A cognitive analysis of problems of comprehension in a learning of mathematics. Educational Studies in Mathematics. New York, Vol. 61, p. 103-131, 2006.

DUVAL, R.. Semiósis e pensamento humano: Registros semióticos e aprendizagens intelectuais (Fascículo I). Tradução de Lênio Fernandes Levy e Marisa Rosâni Abreu da Silveira. São Paulo: Livraria da Física, 2009.

DUVAL, R. Ver e ensinar a matemática de outra forma: entrar no modo matemático de pensar os registros de representações semióticas. Organização Tânia M.M. Campos. Tradução Marlene Alves Dias. São Paulo: PROEM, 2011.

FRANÇA, M. V. D. de. Conceitos fundamentais de Álgebra Linear: uma abordagem integrando geometria dinâmica. Dissertação (Mestrado em Educação Matemática). Pontifícia Universidade Católica de São Paulo. São Paulo: 2007.

GAGNÉ, R. M.. The Conditions of Learning and Theory of Instruction (4th Edition). New York: CBS College Publishing, 1985.

GRANDE, A. L.; BIANCHINI, B. L.. Alguns resultados da análise dos livros didáticos de Álgebra Linear quanto aos registros de representação semiótica e as noções de independência linear. EBRAPEM- Encontro Brasileiro de Estudantes em Educação Matemática. Belo Horizonte, Universidade Federal de Minas Gerais, 2006. Disponível em: http://www.fae.ufmg.br/ebrapem/resumos/10-04res.pdf. Acesso em: 16 abr. 2013. 
HILLEL, J.; DREYFUS, T.. What's a best fit?: Construction of meaning in a linear algebra session. In: KILPATRICK, J.; HOYLES, C.; SKOVSMOSE, O. (Eds.) Meaning in mathematics education. United States of America: Springer, 2005.

KARRER, M.. Articulação entre álgebra linear e geometria: um estudo sobre as transformações lineares na perspectiva dos registros de representação semiótica. Tese (Doutorado em Educação Matemática). Pontifícia Universidade Católica de São Paulo. São Paulo, 2006. Disponível em: http://www.sapientia.pucsp.br//tde busca/arquivo.php?codArquivo=2945. Acesso em 20 set. 2013.

KIERAN, C.; FORMAN, E.; SFARD, A. (Eds.). Bridging the individual and the social. (PME special issue of Educational Studies in Mathematics, 46(1-3)). Dordrecht, The Netherlands: Kluwer Academic, 2001.

LIMA, E. L.. Álgebra Linear.. 2a Ed. Rio de Janeiro: IMPA, 1996.

MACHADO, N. J.. Matemática e língua materna: análise de uma impregnação mútua. 6a ed. São Paulo: Cortez, 2011.

MESSIAS, M. A.V.F.; SÁ, P.F.; FONSECA, R.V.. Um estudo diagnóstico sobre as dificuldades em matrizes. In: Encontro Nacional de Educação de Matemática (ENEM), IX, 2007, Belo Horizonte. Anais... Belo Horizonte: Universidade de Belo Horizonte, 2007.

SIERPINSKA, A.. Discoursing mathematics away. In: KILPATRICK, J.; HOYLES, C.; SKOVSMOSE, O. (Eds.) Meaning in mathematics education. United States of America: Springer, 2005.

STEINBRUCH, A.;WINTERLE, P.. Álgebra Linear. Rio de Janeiro: McGraw-Hill, 1987.

VERGNAUD, G. La théorie des champs conceptuels. Récherches en Didactique des Mathématiques, 10 (23): 133-170, 1990. 